\begin{tabular}{|c|c|}
\hline Title & Monte Carlo analy sis of montmorillonite particle structures and modeling of dissolution rate reduction \\
\hline Author(s) & Terada, Kenichi; Tani, A kiho; Harada, Shusaku; Satoh, Hisao; Hay ashi, Daisuke \\
\hline Citation & $\begin{array}{l}\text { Materials Research Express, 6(3), } 035514 \\
\text { https://doi.org/10.1088/2053-1591/aaf829 }\end{array}$ \\
\hline Issue Date & $2019-03$ \\
\hline Doc URL & http:/hdl.handle.net/2115/6822 \\
\hline Rights & $\begin{array}{l}\text { This is a peer-reviewed, un-copyedited version of an article accepted for publication/published in Materials Research } \\
\text { Express. IoP Publishing Ltd is not responsible for any errors or omissions in this version of the manuscript or any } \\
\text { version derived from it. The Version of Record is available online at https://doi.org/0.1088/2053-1591/aaf829. }\end{array}$ \\
\hline Type & article (author version) \\
\hline File Information & terada_final.pdf \\
\hline
\end{tabular}

Instructions for use 


\title{
Monte Carlo Analysis of Montmorillonite Particle Structures and Modeling of Dissolution Rate Reduction
}

\author{
Kenichi Terada \\ Faculty of Engineering, Hokkaido University, N13-W8, Sapporo, 0608628, Japan \\ Akiho Tani \\ Faculty of Engineering, Hokkaido University, N13-W8, Sapporo, 0608628, Japan \\ Shusaku Harada \\ Faculty of Engineering, Hokkaido University, N13-W8, Sapporo, 0608628, Japan \\ Hisao Satoh
}

Mitsubishi Materials Corporation, 1002-14, Mukohyama, Naka, Ibaraki, 311-0102, Japan

Daisuke Hayashi

Radioactive Waste Management Funding and Research Center, 6-4, Akashicho, Chuo-ku, Tokyo, 104-0044, Japan

The equilibrium structure of montmorillonite clay particles was calculated using Monte Carlo simulation to evaluate the long-term behavior of alteration for bentonite buffer materials in geological repositories of radioactive waste. It has been experimentally observed that the dissolution rate of montmorillonite strongly decreases as the density increases. One of the primary causes of this reduction in dissolution rate is the reduction in reactive surface area when the edges of particles are covered with other particles (physical masking). In this study, we proposed a geometrical model in which the masking area (the edge surface masked by other particles) is calculated from the edge-to-face distance of particles. The effective edge surface area (ESA) from the equilibrium 
structure obtained from Monte Carlo simulation was computed using the masking model. The numerical results of the effective ESA are in good agreement with the experimental measurements of the dissolution rate by atomic force microscopy in a wide range of density conditions. Moreover, we propose a prediction expression of the effective ESA of montmorillonite particles over a wide range of density conditions, which produces physically reasonable predictions.

Keywords: montmorillonite, dissolution rate, equilibrium structure, effective edge surface area

\section{Introduction}

Bentonite clay is known for its good barrier effects in terms of hydraulic, chemical, mechanical and transport processes. Therefore, it has been considered as a possible candidate for the engineered barrier systems of repositories such as geological disposal of radioactive waste. However, it has been observed that in the presence of alkaline groundwater, the barrier effect of bentonite buffer materials is reduced by dissolution, which may occur over a long period of time. This alkaline perturbation is expected to be caused by interaction between groundwater and the cementitious material used in constructing the repository. The degradation in properties is assumed to be dependent on the density of montmorillonite, which is the main component of bentonite buffer materials. It is thus necessary to increase understanding of the mechanisms and kinetics of montmorillonite dissolution.

A number of previous studies have been conducted involving montmorillonite dissolution experiments to provide the necessary evaluation of the bentonite endurance [1]. As a result, there are many kinetic equations explaining dissolution phenomena as a function of chemical affinity, temperature, $\mathrm{pH}$ and mineral surface area [2]. Among these, it is well known that the most intensive parameter is the surface area consisting of dissolution sites such as step edges [3]. Numerical models are becoming more widely used for the prediction of the long-term behavior of the alteration [4-8]. However, these models have not yet completely explained the mechanism of the alteration. One of the primary reasons for this is that the structure of montmorillonite particles cannot be observed in a compacted system (i.e., bentonite buffer materials). Therefore, it remains difficult to make a long-term analytical prediction for the dissolution rate of montmorillonite in a compacted state. 
However, experimental approaches have recently demonstrated that the dissolution rate of montmorillonite drastically decreases as a function of density [9]. This observed reduction may occur when the edge of particles is covered with the other particles and accordingly the reactive surface area is decreased. This mechanism is called physical masking [10]. It is therefore important to understand the thermodynamic equilibrium structure of montmorillonite particles to quantitatively evaluate the dissolution rate.

Several studies have been conducted to reproduce the structure of clay particles [11-13]. Dijkstra et al. performed Monte Carlo analysis of the equilibrium structures of disk-shaped particles in a dispersion system. In the analysis, they used the quadrupole potential, which models the inter-particle interaction of clay particles (e.g., Laponite particles). The quadrupole potential includes the effects of the charge in each particle and the surrounding electric double layer. However, this potential cannot be used to calculate the equilibrium structure at high density.

Some studies have been able to construct a potential that can be applied for high-density conditions of various clay particles [14-18]. In the case of montmorillonite in bentonite buffer materials, it is difficult to obtain an alternative potential that can be used for the high-density conditions. In general, clay particles have an anisotropic shape and the particle structures cannot be observed experimentally in a compacted system. It is assumed that the effect of the electric double layer around the particles may be extremely reduced in a compacted state at high density.

The equilibrium state of anisotropic shaped particles has been studied in various fields. For example, Onsager explained that the disorder-order phase transition of a hard-rod system is caused by the effect of configurational entropy, which minimizes the excluded volume between molecules [19]. Furthermore, the equilibrium structure of many types of anisotropic particles (e.g., cut sphere, infinitely thin hard disk) have been investigated [20-27]. These studies showed that the phase transition of anisotropic particles greatly depends on the shape and the density conditions.

In this study, the equilibrium structure of the compacted montmorillonite in bentonite buffer materials at different densities was simulated using Monte Carlo analysis. The quadrupole potential was used to calculate the equilibrium structure at low density. In addition, the equilibrium structures 
were calculated using the rigid-body potential under high-density conditions. In low- to medium-density cases, the analysis using the quadrupole potential is more realistic than the rigid-body potential in a dispersion system. However, the analysis by rigid-body potential becomes more reasonable at high density because of the limitation of the possible particle configuration. In order to examine the consistency between the analysis using the quadrupole potential and the rigid-body potential, the Monte Carlo analysis by the rigid-body potential was expanded to the low to medium density in this study.

As previously discussed, one of the primary reasons of the dissolution rate reduction for montmorillonite is the physical masking. In this study, the effective edge surface area (ESA) was calculated from the equilibrium structure of disk-shaped particles obtained by Monte Carlo simulation. We estimated the edge surface of particles masked by other particles using a geometric model and calculated the effective ESA, which is the ratio of the unmasked edge surface area to the total ESA. Furthermore, a comparison was made between the experimental results of dissolution rate for montmorillonite and the analytical results of the effective ESA to determine the mechanism of the drastic reduction in the montmorillonite dissolution rate. Based on these results, we propose a expression which gives physically reasonable predictions of the effective ESA of montmorillonite particles.

\section{Calculation method}

\subsection{Monte Carlo analysis}

The Monte Carlo method was used to compute thermodynamic equilibrium structures of disk-shaped particles, which model montmorillonite particles. We assumed a monodispersed system of infinitely thin disk particles. The calculation region was cubic and the periodic boundary conditions were applied in all directions. For the initial configuration, the disk-shaped particles were arranged by setting the position and the normal vector using a random number. We checked the intersection of each particle by geometric calculation and overlap of particles was not allowed. For dense conditions, the particles cannot be arranged uniformly in the calculation region by the 
excluded volume effect. Therefore, we set a limit for the angle of the normal vector in dense cases. The equilibrium structure of particles was determined from several different initial configurations by changing the limiting angle. A Monte Carlo move was performed by changing the position and orientation of each particle based on the Metropolis method [28]. We set the maximum displacement per a translation $\Delta r=0.02 \sigma(\sigma:$ disk diameter) and the maximum variation for each normal vector component $\Delta v=0.02$.

For low- to medium-density conditions, a fixed number of particles $N(N=400)$ was assumed and the volume of calculation region $V$ was changed. In the high-density analysis, the size of calculation region was fixed and $N$ changed depending on the desired density conditions. This was in consideration of the influence of the particle structure in the size of the calculation region. From the results of preliminary calculation, the side length of the calculation region was three times larger than the particle diameter $\sigma$.

\subsection{Inter-particle potential}

Two different potentials were used to calculate the equilibrium structures of montmorillonite particles. For the analysis of particles in a dispersion system under low-density conditions, we used the quadrupole potential proposed by Dijkstra et al. [11] to model anisotropic inter-particle interaction. The quadrupole potential included effects of the charge of disk-shaped particle and the surrounding electric double layer. The potential between two disk-shaped particles was calculated as follows [11]:

$$
v\left(\boldsymbol{R}_{i j}, \boldsymbol{n}_{i}, \boldsymbol{n}_{j}\right)=\frac{3 Q^{2}}{4 \varepsilon\left|\boldsymbol{R}_{i j}\right|^{5}} \Phi(i, j)
$$

where $\boldsymbol{R}_{i j}\left(=\boldsymbol{R}_{i}-\boldsymbol{R}_{j}\right)$ is the relative position vector $\left(\boldsymbol{R}_{i}, \boldsymbol{R}_{j}\right.$ : the position vector at the center of particle $i$ and $j$ ), $\boldsymbol{n}_{i}$ and $\boldsymbol{n}_{j}$ is the unit normal vector of each particle, $\varepsilon$ is a dielectric constant and $Q$ is the quadrupole moment. $Q$ is meant to the strength of the potential included the influence of each particle and the surrounding electric double layer. $\Phi(i, j)$ is the rotational invariant of particle $i$ and $j$ and is defined as follows [29]: 


$$
\begin{aligned}
\Phi(i, j)=1-5 & \cos ^{2} \phi_{i}-5 \cos ^{2} \phi_{j}-15 \cos ^{2} \phi_{i} \cos ^{2} \phi_{j} \\
& +2\left[\sin \phi_{i} \sin \phi_{j} \cos \left(\varphi_{i}-\varphi_{j}\right)-4 \cos \phi_{i} \cos \phi_{j}\right]^{2}
\end{aligned}
$$

where $\phi_{i}, \varphi_{i}$ and $\phi_{j}, \varphi_{j}$ are the polar angles where the polar axis is chosen as $\boldsymbol{R}_{i j}$ as shown in Fig. $1(\mathrm{a})$.

Due to the model limitation, the quadrupole potential cannot be used under dense conditions in which the interference of electric double layers occurs. In this study, the analysis by the quadrupole potential was performed under density conditions less than the nondimensional density $N \sigma^{3} / V=5.5$, which corresponds to the dry density of montmorillonite particle $\rho_{b} \sim 0.02 \mathrm{Mg} / \mathrm{m}^{3}$. In the Monte Carlo analysis, the quadrupole potential $Q$ is set as the nondimensional potential $Q^{2} / \varepsilon k_{B} T \sigma^{5}$. We set the nondimensional potential $Q^{2} / \varepsilon k_{B} T \sigma^{5}=0.1$. This value is close to that in the previous analysis by Dijkstra et al. [11] in which they assumed Laponite particles $(\sigma=25 \mathrm{~nm})$ dispersed in water for about $10^{-4} \mathrm{M}$ salt concentration.

In contrast, the structural analysis by the rigid-body potential was conducted for the high-density conditions as compacted states. The analysis by the rigid-body potential is equivalent to the condition of $Q^{2} / \varepsilon k_{B} T \sigma^{5}=0$ in the analysis by the quadrupole potential. Hence, this analysis could be regarded to represent high-temperature conditions (large $k_{B} T$ ) in terms of the inter-particle potential. In fact, the inter-particle potential may be reduced effectively because of the overlapping of the electric double layer in the compacted states. As mentioned above, the analysis using the quadrupole potential can only be performed under dilute conditions. The analysis by the rigid-body potential was also performed from low- to medium-density conditions and consistency was checked between the two sets of results.

In the Monte Carlo analysis with the rigid-body potential, the intersection of each particle was checked by a geometric calculation. If the particles were overlapped, excessive potential energy was given. For the analysis of the quadrupole potential, the equilibrium structure of the disk-shaped particles was determined whether the total potential energy of the system reached a constant value. However, we could not monitor the total potential energy of the rigid body. Therefore, we quantified 
the pore structure inside the particle and the equilibrium structure was determined whether the pore characteristics (number, size and shape) reached a constant value.

\subsection{Masking model}

A geometric masking model was proposed for the estimation of the effective ESA. In the model, the edge area masked by other particles determined whether or not the vertical distance from the edge point to the surface of other particle was less than the arbitrary value (masking parameter $\delta$ ).

As shown in Fig. 1(b), we consider two disks where $\boldsymbol{p}=\left(p_{x}, p_{y}, p_{z}\right)$ and $\boldsymbol{q}=\left(q_{x}, q_{y}, q_{z}\right)$ are the central position vector, $\boldsymbol{m}=\left(m_{x}, m_{y}, m_{z}\right)$ and $\boldsymbol{n}=\left(n_{x}, n_{y}, n_{z}\right)$ are the normal vector of each disk, respectively. The equation of a plane including each disk is described as follows:

$$
\begin{gathered}
m_{x} x+m_{y} y+m_{z} z=m_{x} p_{x}+m_{y} p_{y}+m_{z} p_{z} \\
n_{x} x+n_{y} y+n_{z} z=n_{x} q_{x}+n_{y} q_{y}+n_{z} q_{z}
\end{gathered}
$$

The arbitrary vector $\boldsymbol{k}=\left(k_{x}, k_{y}, k_{z}\right)$ is defined from the center of the disk $\boldsymbol{p}$ to the edge. For example, if $x$ component of $\boldsymbol{k}$ is set to $0, \boldsymbol{k}$ is expressed using the position vector $\boldsymbol{m}=\left(m_{x}, m_{y}, m_{z}\right)$ as follows:

$$
\left(k_{x}, k_{y}, k_{z}\right)=\left(0,-\frac{m_{z}}{m_{y}} \frac{1}{\sqrt{1+m_{z}^{2} / m_{y}^{2}}}, \frac{1}{\sqrt{1+m_{z}^{2} / m_{y}^{2}}}\right)
$$

Furthermore, the vector $\boldsymbol{k}$ was rotated by small angle $\Delta \varphi$ on the edge of disk $\boldsymbol{p}$. When the rotation angle reaches $\varphi$, the rotated vector $\boldsymbol{u}=\left(u_{x}, u_{y}, u_{z}\right)$ is expressed as follows:

$$
\begin{aligned}
& u_{x}=\frac{\sigma}{2}\left[k_{x} \cos \varphi+\left(m_{y} k_{z}-m_{z} k_{y}\right) \sin \varphi\right] \\
& u_{y}=\frac{\sigma}{2}\left[k_{y} \cos \varphi+\left(m_{z} k_{x}-m_{x} k_{z}\right) \sin \varphi\right] \\
& u_{z}=\frac{\sigma}{2}\left[k_{z} \cos \varphi+\left(m_{x} k_{y}-m_{y} k_{z}\right) \sin \varphi\right]
\end{aligned}
$$

The position vector of a foot of a perpendicular lowered from the edge point to the plane $\boldsymbol{n}=\left(n_{x}, n_{y}\right.$, $\left.n_{z}\right)$ is represented by $\boldsymbol{w}=\left(w_{x}, w_{y}, w_{z}\right)$ described as:

$$
w_{x}=u_{x}+n_{x}\left(n_{x} q_{x}+n_{y} q_{y}+n_{z} q_{z}-n_{x} u_{x}-n_{y} u_{y}-n_{z} u_{z}\right)
$$




$$
\begin{aligned}
& w_{y}=u_{y}+n_{y}\left(n_{x} q_{x}+n_{y} q_{y}+n_{z} q_{z}-n_{x} u_{x}-n_{y} u_{y}-n_{z} u_{z}\right) \\
& w_{z}=u_{z}+n_{z}\left(n_{x} q_{x}+n_{y} q_{y}+n_{z} q_{z}-n_{x} u_{x}-n_{y} u_{y}-n_{z} u_{z}\right)
\end{aligned}
$$

If the distance between the position $\boldsymbol{w}$ and the center of disk $\boldsymbol{q}$ is smaller than the radius of the disk $\sigma / 2$, a foot of perpendicular line exists on the surface of disk $\boldsymbol{q}$. In this case, the vertical distance from the edge point to the surface of disk (thick line in Fig. 1(b)) is calculated. If the distance is less than the masking parameter $\delta$, the edge point is determined to be masked by other particles.

In this study, the total ESA, which is not masked by other particles per unit mass, was defined as the effective ESA. As mentioned previously, the Monte Carlo simulation was performed using infinitely thin disk-shaped particles, while actual montmorillonite particles have a finite thickness. Therefore, the effective ESA was calculated from simulation results under the assumption of finite thickness of particles $d=1 \mathrm{~nm}$. Similarly, the dry density of montmorillonite was calculated from the nondimensional density $N \sigma^{3} / V$ under the assumption that each particle had a thickness $d=1 \mathrm{~nm}$. The obtained results of the effective ESA on various density conditions were compared with those obtained from the experimental results of the montmorillonite dissolution rate by atomic force microscopy (AFM).

\section{Results and discussion}

\subsection{Equilibrium structure by quadrupole potential and rigid-body potential}

Figure 2 shows the equilibrium structure of infinitely thin disk-shaped particles, which models montmorillonite particles in a wide range of nondimensional density $N \sigma^{3} / V$ obtained from the Monte Carlo simulation. Figure 2(a) shows the numerical results using the quadrupole potential for the nondimensional density $N \sigma^{3} / V=0.25-5.0$. The quadrupole potential expresses the anisotropic interaction of disk-shaped particles, which includes the effect of the electric double layer and the charge of each particle. In contrast, Fig. 2(b) shows the results using the rigid-body potential in a wide density range $\left(N \sigma^{3} / V=0.25-170\right)$. 
Figure 3 also shows the close-up images of equilibrium structure by the quadrupole potential and the rigid-body potential under low-density conditions (we put the thickness of disk particles in the images, whereas infinitely-thin particles are assumed in the simulation). As can be seen in Fig. 3, the particles with the quadrupole potential make aggregates with the edge-to-face structure ("house of cards" structure), which is typically observed in clay soil [11]. In contrast, the particles are distributed isotropically in the equilibrium structure with rigid-body potential under very low-density conditions $\left(N \sigma^{3} / V=0.25\right)$.

However, under moderate-density conditions $\left(N \sigma^{3} / V=5.0\right)$, the particles are aligned in one direction and the qualitative difference of structure cannot be observed in both results. This is because the possible state of disk-shaped particles is limited because of the configurational limitation with the increase of the density. As shown in Fig. 2, in the equilibrium structure with rigid-body potential for $N \sigma^{3} / V=30$, almost all of the particles are aligned in one direction. Under such dense conditions, the possible states of the particles would be extremely decreased regardless of the type of inter-particle potential.

\subsection{Phase transition of disk-shaped particles}

It is well-known that the structural changes of anisotropic-shaped particles with the density increase are caused by the configurational limitation of particles. The pioneer work on the phase transition of non-spherical hard molecules was done by Onsager [19], in which disorder-order phase transition was explained from the excluded volume effect. Furthermore, many studies have been conducted on the phase transition of various-shaped particles [20-27,30-32]. For example, Eppenga and Frenkel investigated the phase transition of infinitely thin disk-particles, which is similar to our study [20].

In order to understand how the structures with the quadrupole potential are close to those with the rigid-body potential with the increase of the density, we examined both structural changes quantitatively. Figure 4 shows the nematic order parameter $S$ of the equilibrium structures with both potentials, which is calculated as follows: 


$$
S=\frac{1}{N}\left\langle\sum_{i=1}^{N}\left(\frac{3}{2}\left(n \cdot \theta_{i}\right)^{2}-\frac{1}{2}\right)\right\rangle
$$

where $n$ is the nematic director and $\theta_{l}$ is the orientation angle of each particle. $S=1$ indicates that all particles are aligned in one direction (nematic phase), while $S=0$ if the particles are randomly oriented (isotropic phase).

Nevertheless, the equilibrium structures with the quadrupole and the rigid-body potentials were significantly different (see Fig. 3) and the nematic order parameters with both potentials are almost zero from low to medium density conditions $N \sigma^{3} / V<4.0$. This is because the structures with rigid-body potential were almost uniform and isotropic, whereas the structures with quadrupole potential made aggregates and the orientation of particles was locally isotropic in each "house of cards" aggregate.

From the nondimensional density $N \sigma^{3} / V \sim 4.0$, the nematic order parameter $S$ with both potentials suddenly increased. This is known as the transition from isotropic to nematic phase (IN transition), i.e., the aligned structure of particles is entropically favored with increasing the density. Eppenga and Frenkel reported that IN transition of infinitely thin disk particles occurs at nondimensional density $N \sigma^{3} / V \sim 4.0[20]$. Our results using rigid-body potential agree with their results.

As mentioned above, the quadrupole potential cannot be used under dense conditions because of the limitations of the model and therefore we only examined the equilibrium structure for the nondimensional density less than $N \sigma^{3} / V \sim 5.0$. As shown in Fig. 4, the structures with quadrupole potential showed a similar tendency to those with rigid-body potential and the nematic order parameter suddenly increased around $N \sigma^{3} / V \sim 4.0$. This implies that the aggregates no longer make the "house of cards" structures and the particles form a chain of the edge-to-face structures as the density increases. The nematic order parameter of both potentials had almost the same values for the nondimensional density $N \sigma^{3} / V>5.0$. Therefore, the structures were almost the same regardless of the inter-particle potential when the density was larger than that in the IN transition. 
As previously mentioned, the nondimensional potential $Q^{2} / \varepsilon k_{B} T \sigma^{5}=0.1$ was assumed for the analysis by quadrupole potential, where $Q$ expresses the strength of inter-particle force and $T$ is the absolute temperature. Conversely, the analysis by the rigid-body potential was equivalent to the condition of the nondimensional potential $Q^{2} / \varepsilon k_{B} T \sigma^{5}=0$. In other words, the analysis by the rigid-body potential and the quadrupole potential can be regarded as the high and low temperature conditions, respectively. As described above, under the conditions for the nondimensional density $N \sigma^{3} / V=0-4.0$, the qualitative difference of structure can be observed in both analytical results (Fig. 3). Hence, the equilibrium structure of particles from low to medium density (before IN transition) depends on the temperature. However, from medium to high density (after IN transition), the difference of the equilibrium structure between the two potentials was not significant. Therefore, the equilibrium structure of particles for high density was mostly determined by density conditions regardless of the temperature. The above results suggest that the equilibrium structure of montmorillonite particles under high density conditions can be roughly evaluated by the Monte Carlo analysis using the rigid-body potential.

\subsection{Evaluation of masking area}

We evaluated the effective ESA from the equilibrium structure of particles obtained by Monte Carlo analysis using the quadrupole potential and the rigid-body potential. In the calculation of the effective ESA, we identified the masking areas using the masking model described in Section 2.3. The masking parameter $\delta$ was normalized by particle diameter $\sigma$. In this study, we set $\delta / \sigma=0.01$, $0.02,0.04$ and 0.08 . These values are equivalent to the vertical distance from edge to surface of particles 4.5, 9, 18 and $36 \mathrm{~nm}$ respectively, which are converted by physical properties of the montmorillonite particle $(\sigma=450 \mathrm{~nm})$.

Figure 5 shows the visualized results of masking areas of the equilibrium structure of disk-shaped particles obtained from Monte Carlo analysis with masking parameter $\delta / \sigma=0.04$. In the figure, the masking areas are indicated by the red lines. Under very low-density conditions $\left(N \sigma^{3} / V=0.25\right.$ or 1.0), there are few masking areas in the structure based on the rigid-body potential, whereas several 
masking areas can be seen in the structure from the quadrupole potential. This is because edge-to-face aggregates are made in the quadrupole analysis, whereas the particles with rigid-body potential are distributed uniformly.

The masking area of both structures increase with the density and there is no difference between the two under medium-density conditions $\left(N \sigma^{3} / V=5.0\right)$. This is because the particle structures by both potentials were similar because of the configurational limitation by the excluded volume effect as previously described. Furthermore, almost all of the edge area is identified as masking area in the structures with rigid-body potential at higher density $\left(N \sigma^{3} / V=30-280\right)$. These results are qualitatively consistent with a rapid decrease of dissolution rate in the packing state of montmorillonite particles.

\subsection{Effective ESA}

The effective ESA, which is unmasking edge area per unit mass, was calculated from the results on masking area shown above. Basically, the masking area vanishes under very dilute conditions since the particles do not interfere with each other. Hence, the effective ESA at a dilution limit can be described as follows:

$$
(\text { Effective ESA })=\frac{N \pi \sigma d}{N \rho_{p} \frac{\pi \sigma^{2}}{4} d}=\frac{4}{\rho_{p} \sigma}
$$

where $N$ is the number of particles, $\rho_{p}$ is the particle density and $d$ is the thickness of the particle. By substituting the physical property of the montmorillonite particle $\left(\rho_{p}=2400 \mathrm{~kg} / \mathrm{m}^{3}, \sigma=450 \mathrm{~nm}, d=\right.$ $1 \mathrm{~nm}$ ) into Eq. (13), the theoretical value of the effective ESA at a dilution limit was obtained as 3.7 $\mathrm{m}^{2} / \mathrm{g}$.

Figure 6 shows the relationship between the $\rho_{b}$ and the effective ESA with various nondimensional masking parameters $\delta / \sigma$. The figure shows the effective ESA calculated from both structures by the quadrupole and rigid-body potentials. However, the results by the quadrupole potential are only plotted at dilute conditions $\left(\rho_{b}<0.02 \mathrm{Mg} / \mathrm{m}^{3}\right)$ because of the limitation of the potential. 
It is found from Fig. 6 that the effective ESA drastically decreases with the increase of $\rho_{b}$ especially in the case of a large $\delta / \sigma$. This is due to increase in the edge area masked by other particles. Fig. 6(b) shows an enlarged view of the effective ESA from low to medium density conditions. At a low-density limit, the effective ESA by the rigid-body potential smoothly approaches to the theoretical value $3.7 \mathrm{~m}^{2} / \mathrm{g}$ given in Eq. (13), while the results by the quadrupole potential show discontinuous change. This is because the particles make aggregates with edge-to-face structures even in the dilute case. However, as the density increases, the effective ESA by the quadrupole and rigid-body potential are almost consistent and are connected smoothly at $\rho_{b}=0.02-0.03 \mathrm{Mg} / \mathrm{m}^{3}$ under all conditions of the $\delta / \sigma$. These results are consistent for equilibrium structures of both potentials for the low to medium density conditions shown in Fig. 2. Therefore, the Monte Carlo analysis using the rigid-body potential under the high-density conditions seems to be reasonable, at least for the purpose of the evaluation of the effective ESA.

\subsection{Comparison with analytical and experimental results}

It is generally observed that montmorillonite dissolution occurs at edge faces. Therefore, the dissolution rate can be obtained by the size decreases from the starting materials (i.e., the difference in particle diameter). We carried out a dissolution experiment of montmorillonite (Kunipia P, Kunimine Industry Co.) under various gel densities from $\rho_{b}$ of 0.005 to $0.205 \mathrm{Mg} / \mathrm{m}^{3}$. Particle measurement was performed on mono-dispersed montmorillonite mounted on mica substrates with AFM (Bulker, Dimension 3100). Obtained images over two hundred counts were analyzed to be statistical data of the particle size distributions giving representative sizes as the median values. The dissolution rate $\left(\mathrm{mol} / \mathrm{m}^{2} / \mathrm{s}\right)$ was obtained from the volume change $\left(\mathrm{m}^{3}\right)$ from the initial montmorillonite divided by the molar volume $\left(2.728 \times 10^{-6} \mathrm{~m}^{3} / \mathrm{mol}\right)$ and reaction time (s). Moreover, the nondimensional dissolution rate was calculated by normalizing the dissolution rate for each density to the measured value for the most diluted condition (the details of experimental conditions are given in the supplementary table). 
The nondimensional dissolution rate corresponds to the reactive surface area ratio ESA/ESA. The effective ESA was calculated by multiplying ESA/ESA 0 and the theoretical value of the effective ESA for dilute limit $\left(3.7 \mathrm{~m}^{2} / \mathrm{g}\right)$.

Figure 7 shows the relationship between the numerical results of the effective ESA and normalized results obtained from the experimental measurements of dissolution rate and theoretical formula of the effective ESA, which is described later. It is found from Fig.7 that the numerical results of the effective ESA are quantitatively in good agreement with those obtained from the experimental measurement. The experimental results show that the effective ESA decreased drastically under dilute conditions $\rho_{b}<0.1 \mathrm{Mg} / \mathrm{m}^{3}$. These density conditions are equivalent to nondimensional density $N \sigma^{3} / V<30$ in the Monte Carlo analysis. The particles in these conditions were aligned in one direction, which causes the masking of other particles.

Figure 7(b) shows a close-up plot of the effective ESA from low- to medium-density conditions. The effective ESA obtained from the experiment is close to the analytical results using the masking parameter $\delta / \sigma=0.08$. This value is equivalent to the masking distance $\delta=36 \mathrm{~nm}$ for montmorillonite particles $(\sigma=450 \mathrm{~nm})$ and it seems to be large from a physical point of view. The cause of such a large masking distance remains unclear. However, the numerical results of the effective ESA computed from equilibrium structure obtained by Monte Carlo analysis represent well the change in reactive surface area obtained from the AFM experiment. Therefore, the major reason for dissolution rate reduction is that the edges of particles are covered with the other particles and consequently the reactive surface area is reduced (physical masking).

\subsection{Formulation of the effective ESA}

We have provided a physical explanation on the rapid decrease of the effective ESA under high-density conditions. The increase of masking area results from the approach of each particle with density increase. Paineau et al. experimentally indicated that the change in the inter-particle distance of clay particles [33]. The relationship between the average inter-particle distance of clay and the dry density can be explained by a simple model considering the occupancy volume of 
particles. As shown in Fig. 3, the particle structure changes with increasing the density (IN transition). Therefore, the size of the occupancy volume of each particle may change under dilute and dense conditions.

Under the low-density conditions, the particle distributes isotropically without any constraint of other particles. In this case, the occupancy volume is roughly illustrated as a cube with the inter-particle distance $l_{c}$. As a result, the number density of particle $n(=N / V)$ is proportional to $l_{c}{ }^{-3}$. Consequently, the relationship between the $\rho_{b}$ and the average inter-particle distance $l_{c}$ at low density can be described as follows:

$$
l_{c} \sim n^{-1 / 3} \propto \rho_{b}^{-1 / 3} .
$$

In contrast, the particles are aligned in one direction at high density as shown in Fig. 2. In this case, the occupancy volume should be assumed as a cylindrical column with diameter $\sigma$ and the height $l_{c}$ (inter-particle distance). As a result, the relationship between the $\rho_{b}$ and the inter-particle distance $l_{c}$ in the high density can be described as:

$$
l_{c} \sim \frac{4}{\pi \sigma^{2}} n^{-1} \propto \rho_{b}^{-1}
$$

In summary, the average inter-particle distance of disk-shaped particles is proportional to a $\rho_{b}{ }^{-1 / 3}$ at low density and $\rho_{b}{ }^{-1}$ at high density, respectively.

If we assume that the dependence of effective ESA on the density is similar to that of inter-particle distance, we can express the relationship between the effective ESA and the $\rho_{b}$ as the following expression:

$$
(\text { Effective ESA })=\frac{\mathrm{ESA}_{0}}{\left(\frac{\rho_{b}}{\rho_{b}^{*}}\right)^{\frac{1}{3}}+\left(\frac{\rho_{b}}{\rho_{b}^{*}}\right)}
$$

where $\mathrm{ESA}_{0}$ is the effective ESA for a limiting dilution condition, $\rho_{b}$ is the dry density and $\rho_{b}{ }^{*}$ is the parameter which determines a change point of slope of the effective ESA.

The parameter $\rho_{b}{ }^{*}$ should be decided in conjunction with structural change of particles. As discussed in Section 3.2, the phase transition from isotropic to nematic phase (IN transition) for the equilibrium structure of disk-shaped particles may occur around the nondimensional density $N \sigma^{3} / V$ 
$=4.0$. It is reasonable to assume that $\rho_{b}{ }^{*}$ is the density at which IN transition occurs $\left(\rho_{b}{ }^{*}=0.0168\right.$ $\mathrm{Mg} / \mathrm{m}^{3}$, which is calculated from the physical properties of montmorillonite particles).

Figure 7 shows the comparison of the model results of effective ESA calculated from Eq. (16) with the numerical and experimental results. The model results of the effective ESA exceed the theoretical dilution limit described in Eq. (13) and it goes to infinity as $\rho_{b}$ approaches 0 . Therefore, we set the effective ESA as $\mathrm{ESA}_{0}=3.7 \mathrm{~m}^{2} / \mathrm{g}$ if it exceeds $\mathrm{ESA}_{0}$. Figure 7 indicates that the model results show good agreement with the numerical results using the Monte Carlo method $(\delta / \sigma=0.08)$ and experimental results by AFM.

In summary, we propose the following expression for the relationship between the effective ESA and the dry density of disk-shaped particles.

$$
(\text { Effective ESA })=\left\{\begin{array}{cl}
\mathrm{ESA}_{0} & \left(0 \leq \rho_{b} \leq \rho_{b 0}\right) \\
\frac{\mathrm{ESA}_{0}}{\left(\frac{\rho_{b}}{\rho_{b}^{*}}\right)^{\frac{1}{3}}+\left(\frac{\rho_{b}}{\rho_{b}^{*}}\right)} & \left(\rho_{b 0} \leq \rho_{b}\right)
\end{array}\right.
$$

where $\rho_{b}$ is the dry density, $\rho_{b}{ }^{*}$ is the dry density at which the IN transition occurs, ESA $\mathrm{A}_{0}$ is the effective ESA for a dilution limit and $\rho_{b 0}$ is a lower-limit density of the model. Using the physical properties of montmorillonite particles, the parameters in Eq. (17) are $\rho_{b}{ }^{*}=0.0168 \mathrm{Mg} / \mathrm{m}^{3}, \mathrm{ESA}_{0}=$ $3.7 \mathrm{~m}^{2} / \mathrm{g}$ and $\rho_{b 0}=0.00534 \mathrm{Mg} / \mathrm{m}^{3}$, respectively.

\section{Summary and conclusion}

In order to examine the mechanisms behind the dissolution rate reduction of montmorillonite with the increase of the density, Monte Carlo analysis of infinitely thin disk-shaped particle was performed using two different inter-particle potentials. The equilibrium structure of the particles was calculated for a wide range of density conditions. The quadrupole potential was used for inter-particle interaction in a dispersion system at low density, whereas the rigid-body potential was used from low- to high-density conditions. We examined the particle structures obtained by both 
potentials qualitatively and quantitatively and checked the consistency of these structures under medium-density conditions.

The Monte Carlo analysis results show that the equilibrium structures of particles were different for the two potentials at low density. The particles with the rigid-body potential were distributed uniformly and isotropically, while the particles with quadrupole potential made aggregates with edge-to-face structure. However, under moderate-density conditions in which the excluded volume of particles overlap each other, the difference of particle structures could not be seen in both results. This was caused by the phase transition from isotropic phase to nematic phase (IN transition) and the particle structures with both potentials were gradually aligned in one direction after IN transition.

The equilibrium structure obtained from Monte Carlo analysis using the different potentials indicated that the masking area (the ESA of particles masked by the other particles) could be identified by a geometric masking model and the effective ESA (unmasking edge area per unit mass) was calculated. Although the effective ESA using the rigid-body potential did not coincide with the quadrupole potential at low density, both results connected smoothly under the moderate-density condition. The results of the effective ESA are quantitatively in good agreement with those evaluated from experimental results of the dissolution rate of clay particles. These results imply that the primary reason for the dissolution rate reduction of montmorillonite particle at high density is physical masking, i.e., the edge of particles is covered with the other particles and the reactive surface area is reduced.

We proposed an expression for predicting the effective ESA of disk-shaped particles at any density condition. From the analogy of the dependence of inter-particle distance of disk-shaped particles on the density, we formulated the relationship between the effective ESA and $\rho_{b}$. The proposed expression can express the effective ESA for a wide range of densities. The results of this study can contribute to long-term prediction of the performance of bentonite buffer materials in geological disposal systems. 


\section{Acknowledgments}

This research includes a part of the results of "Advancement of Processing and Disposal Technique for the Geological disposal of TRU Waste (FY2016 and FY2017)" under a grant from the Agency for Natural Resources and Energy (ANRE) in the Ministry of Economy, Trade and Industry (METI) of Japan. 


\section{Reference}

1. Nakayama S., Sakamoto Y., Yamaguchi T., Akai M., Tanaka T., Sato T., Iida Y. Dissolution of montmorillonite in compacted bentonite by highly alkaline aqueous solutions and diffusivity of hydroxide ions. Appl. Clay Sci., 2004;27:53-65.

2. Lasaga, A. C. Kinetic Theory in the Earth Sciences, Princeton Univ. Press, Princeton, NJ. 1998.

3. Lasaga, A. C., Luttge, A. Variation of crystal dissolution rate based on a dissolution stepwave model. Science, 2001;291(5512), 2400-2404.

4. Cama J., Ganor J., Ayora C., Lasaga A. Smectite dissolution kinetics at $80^{\circ} \mathrm{C}$ and $\mathrm{pH} 8.8$. Geochim. Cosmochim. Ac., 2000;64:2701-2717.

5. Rozalén M.L., Huertas F.J., Brady P.V., Cama J., García-Palma S., Linares J. Experimental study of the effect of $\mathrm{pH}$ on the kinetics of montmorillonite dissolution at $25^{\circ} \mathrm{C}$. Geochim. Cosmochim. Ac., 2008;72(17):4224-4253.

6. Bauer A., Berger G. Kaolinite and smectite dissolution rate in high molar $\mathrm{KOH}$ solutions at $35^{\circ}$ and $80^{\circ} \mathrm{C}$. Appl. Geochem., 1998;13(7):905-916.

7. Bauer A., Velde B., Berger G. Kaolinite transformation in high molar KOH solutions. Appl. Geochem. 1998;13(5):619-629.

8. Savage D., Noy D., Mihara M. Modelling the interaction of bentonite with hyperalkaline fluids. Appl. Geochem., 2002;17(3):207-223.

9. Satoh H., Ishii T., Owada H. Dissolution of compacted montmorillonite at hyperalkaline $\mathrm{pH}$ and $70^{\circ} \mathrm{C}$ : in situ VSI and ex situ AFM measurements. Clay Miner., 2013;48(2):285-294.

10. Tournassat C., Steefel C.I., Bourg I.C., Bergaya F. Natural and Engineered Clay Barriers, Vol.6, Elsevier, 2015.

11. Dijkstra M., Hansen, J.P., Madden P.A. Statistical model for the structure and gelation of smectite clay suspensions. Phys. Rev. E, 1997;55(3):3044.

12. Fartaria R., Javid N., Pethrick R.A., Liggat J.J., Sefcik, J., Sweatman M.B. Structure of laponite-styrene precursor dispersions for production of advanced polymer-clay nanocomposites. Soft Matter, 2011;7(19):9157-9166.

13. Ruzicka B., Zaccarelli E., Zulian L., Angelini R., Sztucki M., Moussaïd A., Sciortino F. Observation of empty liquids and equilibrium gels in a colloidal clay. Nat. Mater., 2011;10(1):56-60.

14. Ruzicka B., Zaccarelli E. A fresh look at the Laponite phase diagram. Soft Matter, 2011;7(4): 1268-1286.

15. Fartaria R., Javid N., Pethrick R.A., Liggat J.J., Sefcik J., Sweatman M.B. Structure of 
laponite-styrene precursor dispersions for production of advanced polymer-clay nanocomposites. Soft Matter, 2011;7(19):9157-9166.

16. Jabbari-Farouji S., Weis J.J., Davidson P., Levitz P., Trizac E. Interplay of anisotropy in shape and interactions in charged platelet suspensions. J. Chem. Phys., 2014;141(22):224510.

17. Ebrahimi D., Whittle A.J., Pellenq R.J.M. Mesoscale properties of clay aggregates from potential of mean force representation of interactions between nanoplatelets. J. Chem. Phys., 2014;140(15):154309.

18. Delhorme M., Jönsson B., Labbez C. Monte Carlo simulations of a clay inspired model suspension: the role of rim charge. Soft Matter, 2012;8(37):9691-9704.

19. Onsager L. The effects of shape on the interaction of colloidal particles. Ann. N. Y. Acad. Sci., 1949;51(1): 627-659.

20. Eppenga R., Frenkel D. Monte Carlo study of the isotropic and nematic phases of infinitely thin hard platelets. Mol. Phys., 1984;52(6):1303-1334.

21. Bates M.A., Frenkel D. Infinitely thin disks exhibit a first order nematic-columnar phase transition. Phys. Rev. E, 1998;57(4):4824.

22. Bates M.A., Frenkel D. Nematic-isotropic transition in polydisperse systems of infinitely thin hard platelets. J. Chem. Phys., 1999;110(13):6553-6559.

23. Veerman J.A.C., Frenkel D. Phase behavior of disklike hard-core mesogens. Phys. Rev. A, 1992;45(8):5632.

24. Bolhuis P., Frenkel D. Tracing the phase boundaries of hard spherocylinders. J. Chem. Phys., 1997;106(2):666-687.

25. Wouterse A., Williams S.R., Philipse A.P. Effect of particle shape on the density and microstructure of random packings. J. Phys.: Condens. Matter, 2007;19(40):406215.

26 Guevara-Rodríguez F.D.J., Odriozola G. Hard ellipsoids: Analytically approaching the exact overlap distance. J. Chem. Phys., 2011;135(8):084508.

27. Cheung D.L., Anton L., Allen M.P., Masters A.J., Phillips J., Schmidt M. Structure and stability of isotropic states of hard platelet fluids. Phys. Rev. E, 2008;78(4):041201.

28. Metropolis N., Rosenbluth A.W., Rosenbluth M.N., Teller A.H., Teller E. Equation of state calculations by fast computing machines. J. Chem. Phys., 1953;21(6):1087-1092.

29. Blum L., Torruella A.J. Invariant Expansion for Two - Body Correlations: Thermodynamic Functions, Scattering, and the Ornstein-Zernike Equation. J. Chem. Phys. 1972;56(1):303-310.,

30. Delhorme M., Jönsson B., Labbez C. Gel, glass and nematic states of plate-like particle suspensions: charge anisotropy and size effects. RSC Adv., 2014;4(66):34793-34800. 
31. Marechal M., Cuetos A., Martínez-Haya B., Dijkstra M. Phase behavior of hard colloidal platelets using free energy calculations. J. Chem. Phys., 2011;134(9):094501.

32. Stroobants A., Lekkerkerker H.N.W. Liquid crystal phase transitions in a solution of rodlike and disklike particles. J. Chem. Phys., 1984;88(16):3669-3674.

33. Paineau E., Bihannic I., Baravian C., Philippe A.M., Davidson P., Levitz P., Michot L.J. Aqueous suspensions of natural swelling clay minerals. 1. Structure and electrostatic interactions. Langmuir, 2011;27(9):5562-5573. 


\section{List of Figures}

Fig. 1 (a) Positional relation between two disk-shaped particles. (b) Settings of the masking parameter $\delta$ to calculate the areas that are masked by other particles (masking area). The masking area is defined if the vertical distance from the edge to surface of particles is less than $\delta$.

Fig. 2 Equilibrium structure of infinitely thin disk-shaped particles which model montmorillonite particles by the Monte Carlo method using different potentials.

Fig. 3 Comparison of equilibrium structure based on quadrupole potential and rigid-body potential.

Fig. 4 Evaluation of nematic order parameter $S$ of equilibrium structure of platelet particles by quadrupole potential and rigid body potential for various density conditions.

Fig. 5 Visualization of masking area (red area) of the equilibrium structure of platelet particles calculated by the masking model (masking parameter $\delta / \sigma=0.04)$.

Fig. 6 Effective ESA calculated from the masking model with various masking parameters $\delta / \sigma$.

Fig. 7 Comparison of numerical results of effective ESA, experimental results of dissolution rate and theoretical model under various density conditions.

\section{Supplementary Table}

Table 1 Results of the dissolution experiment of montmorillonite by AFM 


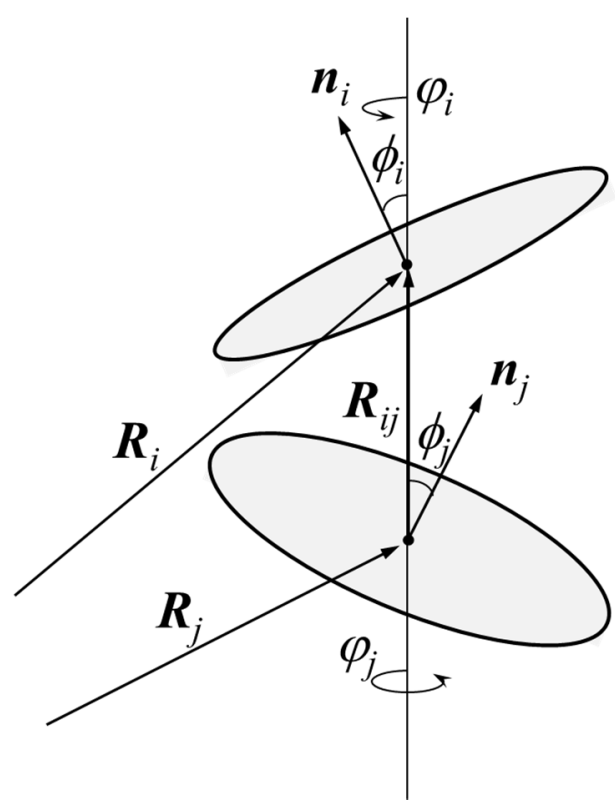

(a)

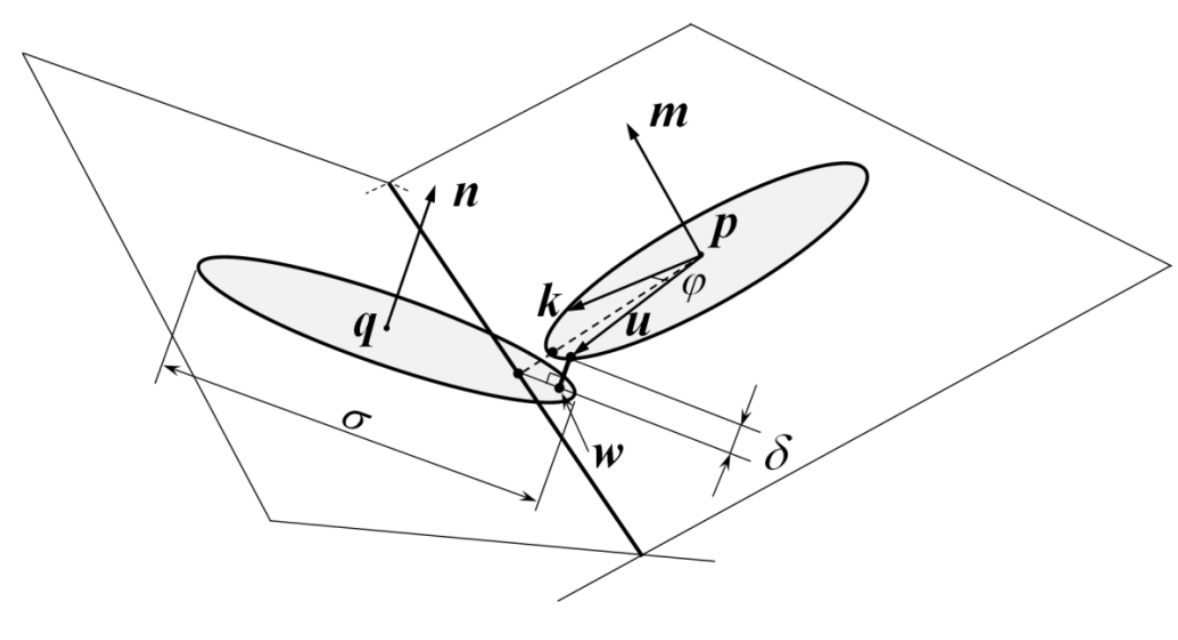

(b)

Fig. 1 (a) Positional relation between two disk-shaped particles. (b) Settings of the masking parameter $\delta$ to calculate the areas that are masked by other particles (masking area). The masking area is defined if the vertical distance from the edge to surface of particles is less than $\delta$. 
(a) quadrupole potential
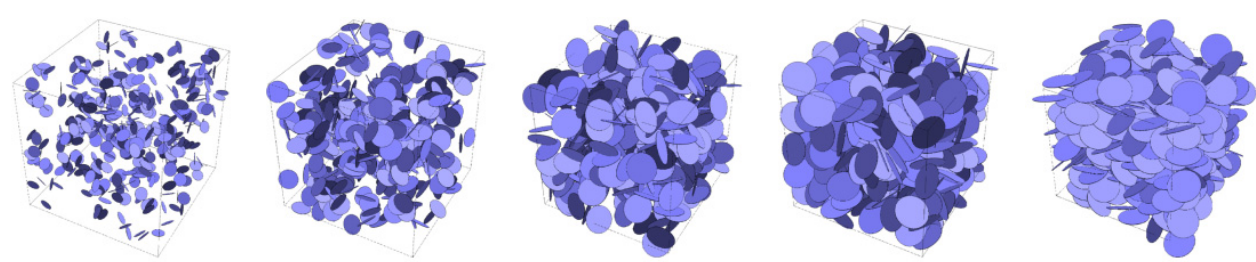

$N \sigma^{3} / V=0.25$

$N \sigma^{3} / V=1.0$

$N \sigma^{3} / V=3.0$

$N \sigma^{3} / V=4.0$

$N \sigma^{3} / V=5.0$

(b) rigid-body potential

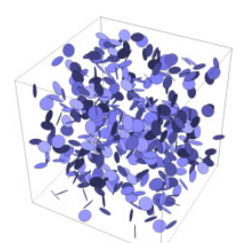

$N \sigma^{3} / V=0.25$

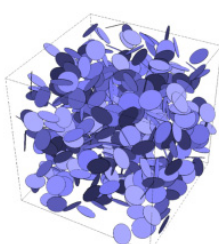

$N \sigma^{3} / V=1.0$

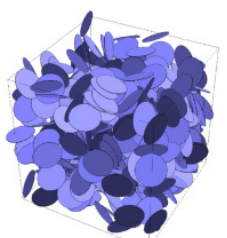

$N \sigma^{3} / V=3.0$

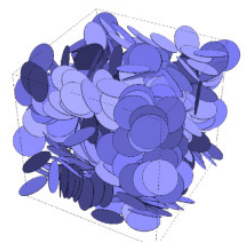

$N \sigma^{3} / V=4.0$

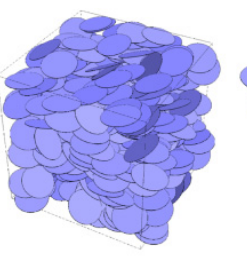

$N \sigma^{3} / V=5.0$

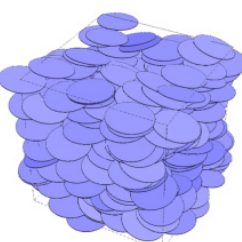

$N \sigma^{3} / V=10$

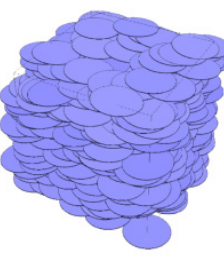

$N \sigma^{3} / V=30$

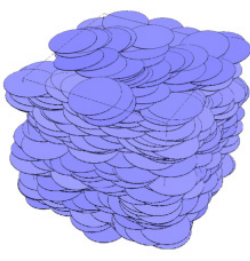

$N \sigma^{3} / V=50$

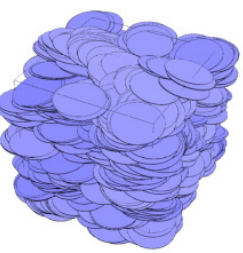

$N \sigma^{3} / V=100$

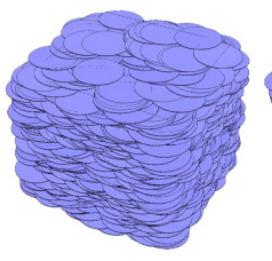

$N \sigma^{3} / V=170 \quad N \sigma^{3} / V=280$

Fig. 2 Equilibrium structure of infinitely thin disk-shaped particles which model montmorillonite particles by the Monte Carlo method using different potentials. 
(a) quadrupole potential

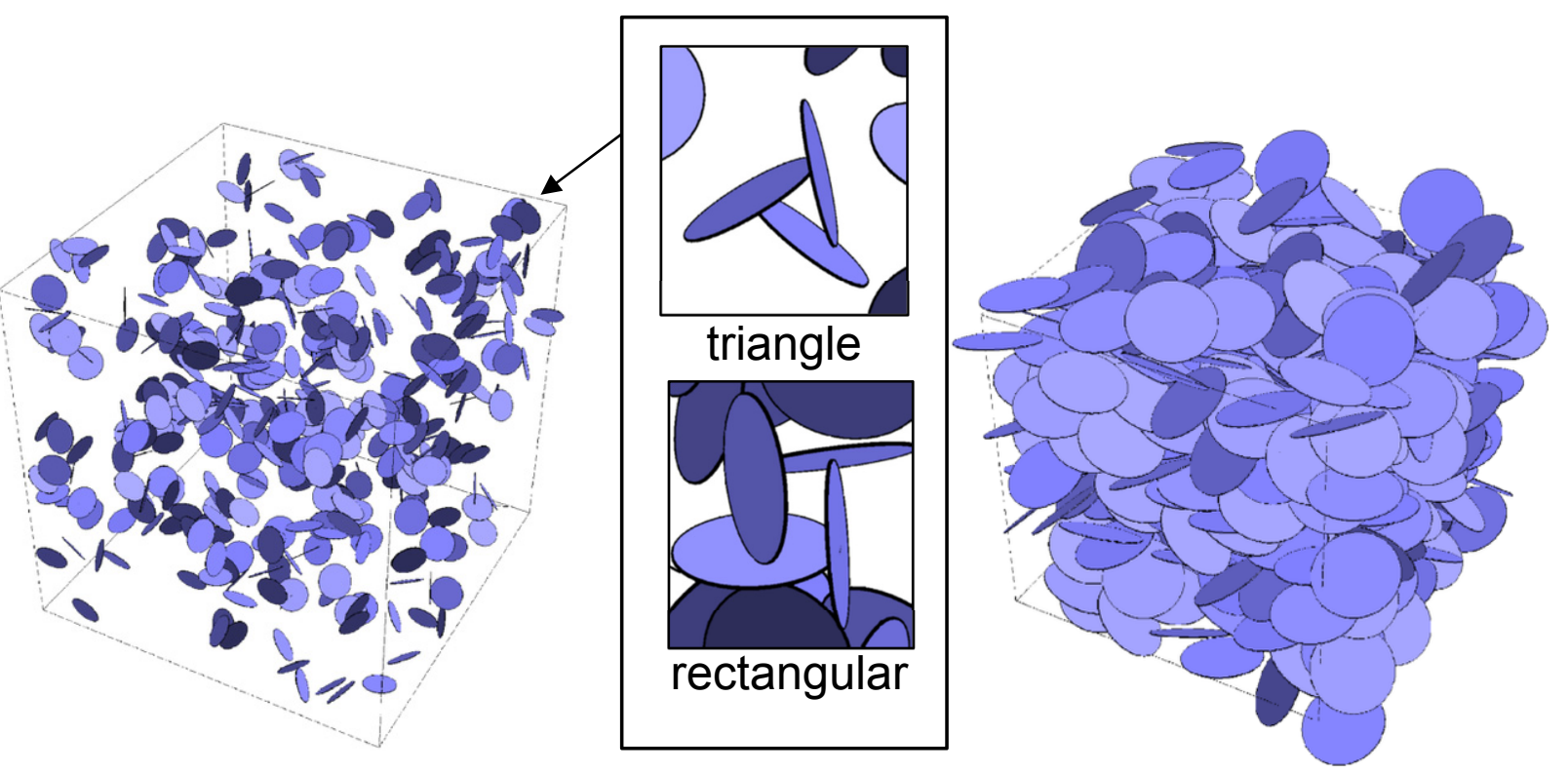

(b) rigid-body potential

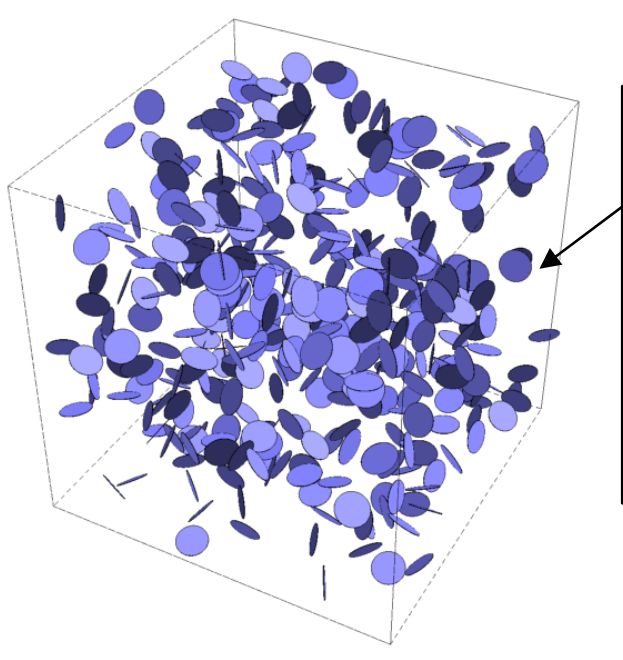

(I) $N \sigma^{3} / V=0.25$
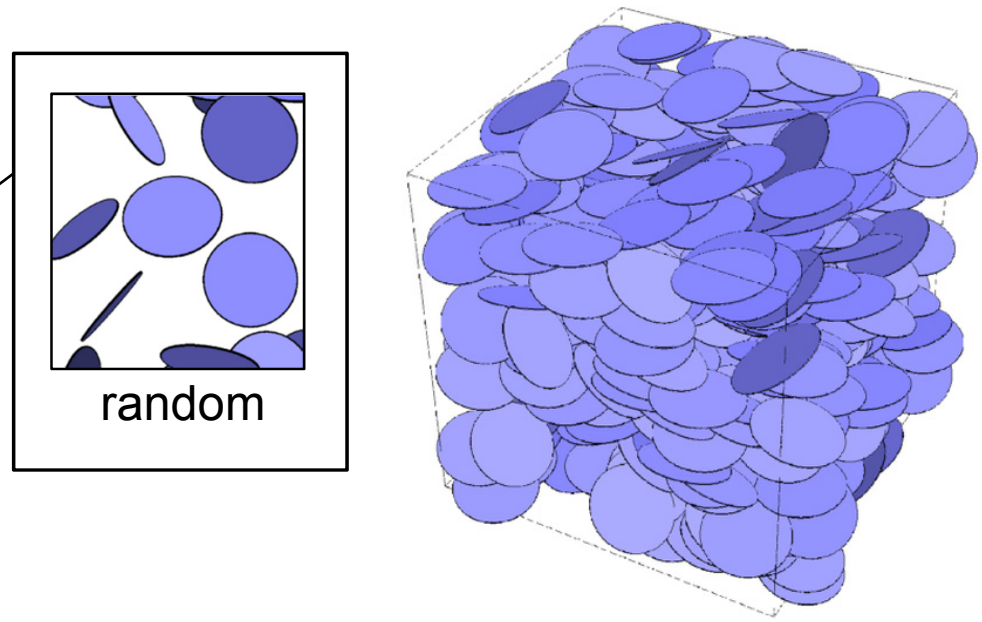

(II) $N \sigma^{3} / V=5.0$

Fig. 3 Comparison of equilibrium structure based on quadrupole potential and rigid-body potential. 


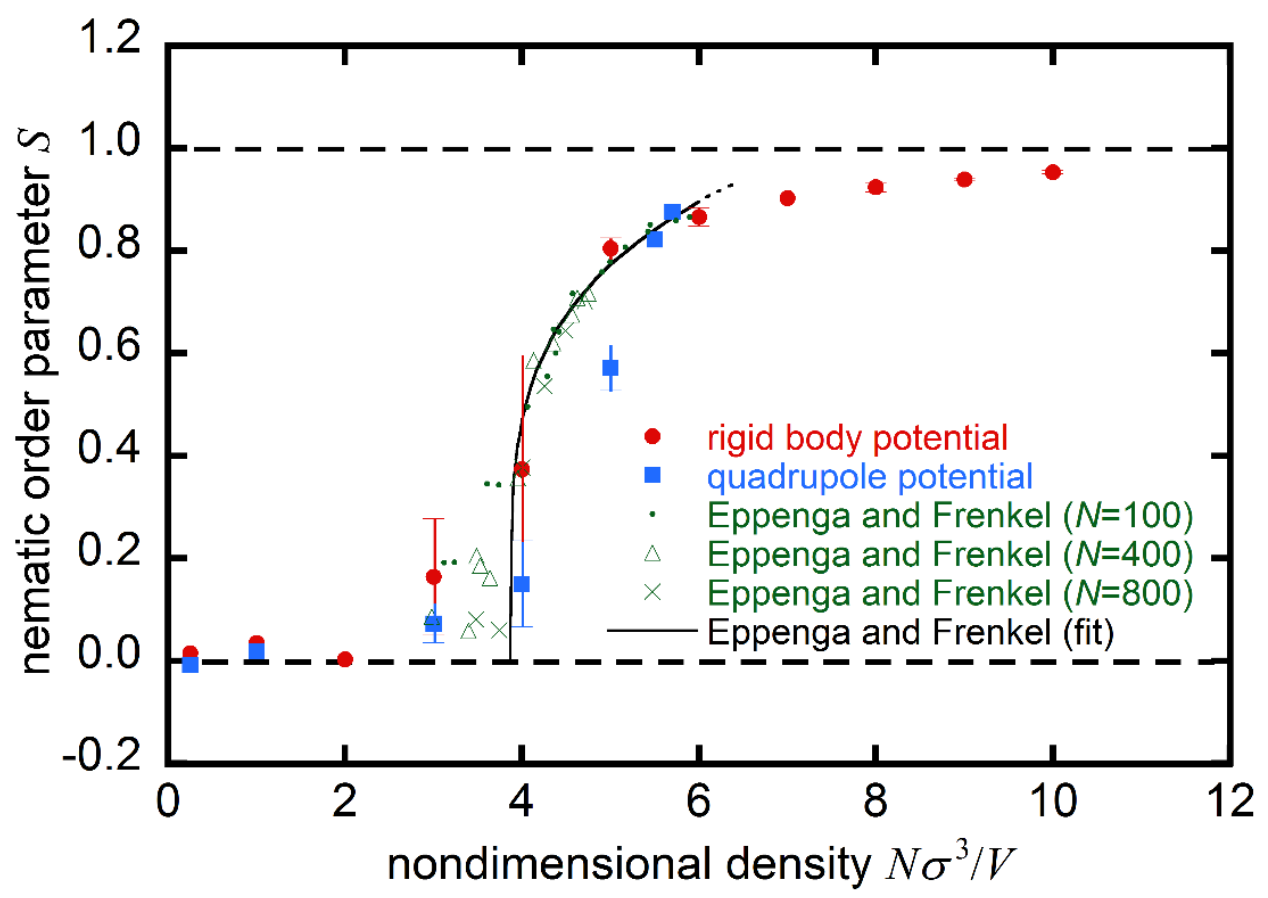

Fig. 4 Evaluation of nematic order parameter $S$ of equilibrium structure of platelet particles by quadrupole potential and rigid-body potential for various density conditions. 
(a) quadrupole potential

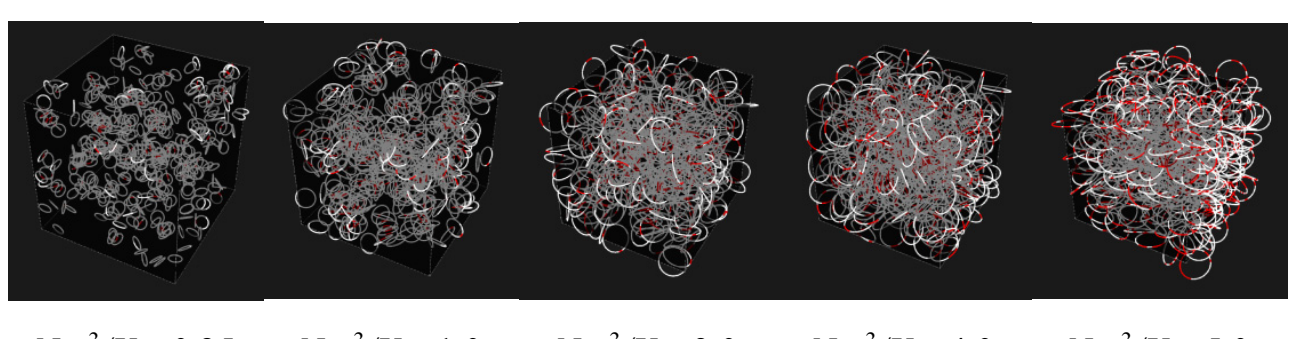

$N \sigma^{3} / V=0.25 \quad N \sigma^{3} / V=1.0 \quad N \sigma^{3} / V=3.0 \quad N \sigma^{3} / V=4.0 \quad N \sigma^{3} / V=5.0$

(b) rigid-body potential

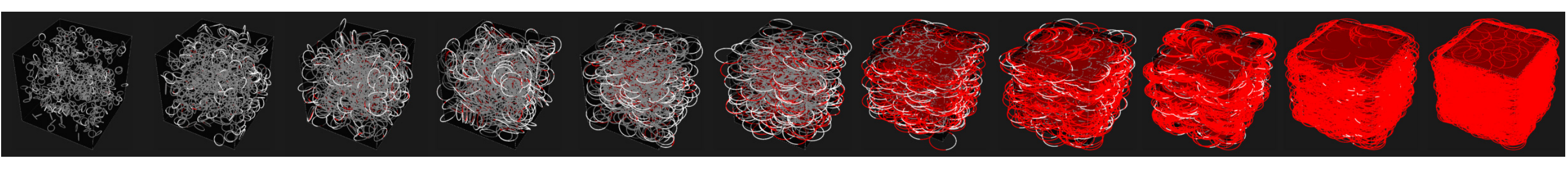

$N \sigma^{3} / V=0.25 \quad N \sigma^{3} / V=1.0 \quad N \sigma^{3} / V=3.0 \quad N \sigma^{3} / V=4.0 \quad N \sigma^{3} / V=5.0 \quad N \sigma^{3} / V=10 \quad N \sigma^{3} / V=30 \quad N \sigma^{3} / V=50 \quad N \sigma^{3} / V=100 \quad N \sigma^{3} / V=170 \quad N \sigma^{3} / V=280$

Fig. 5 Visualization of masking area (red area) of the equilibrium structure of platelet particles calculated by the masking model (masking parameter $\delta / \sigma=$ $0.04)$. 


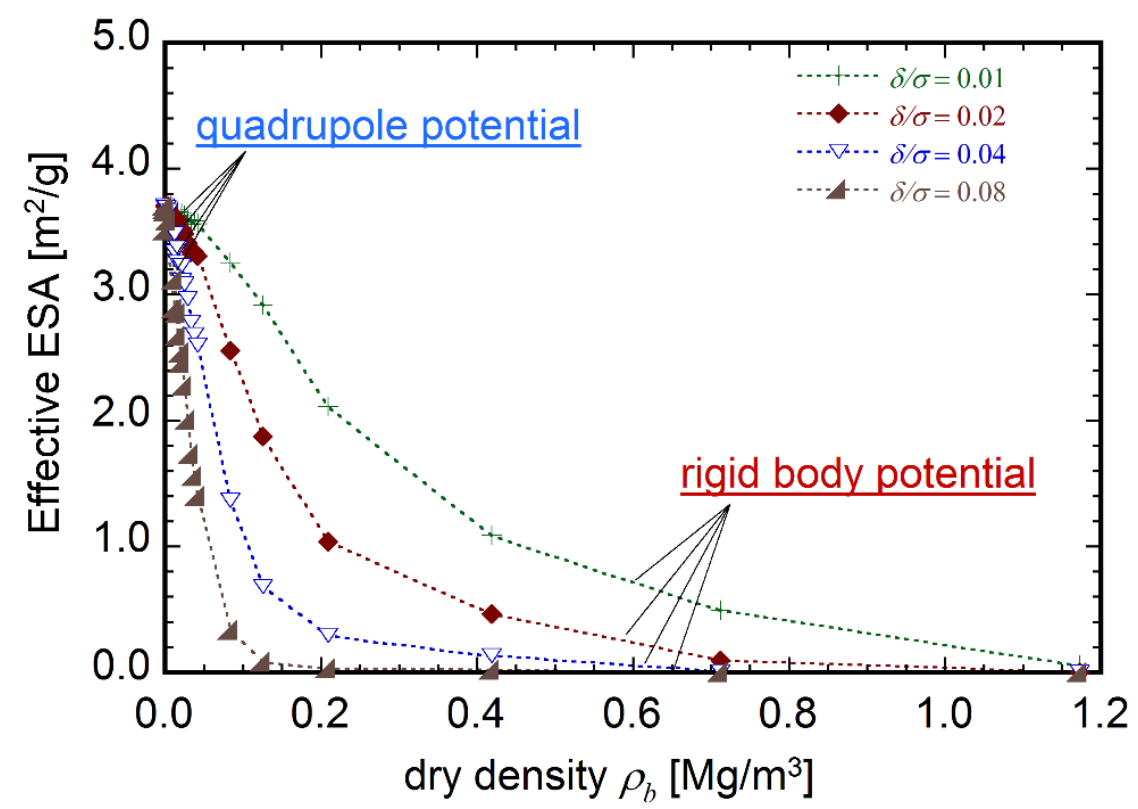

(a) Effective ESA in a wide range of dry densities

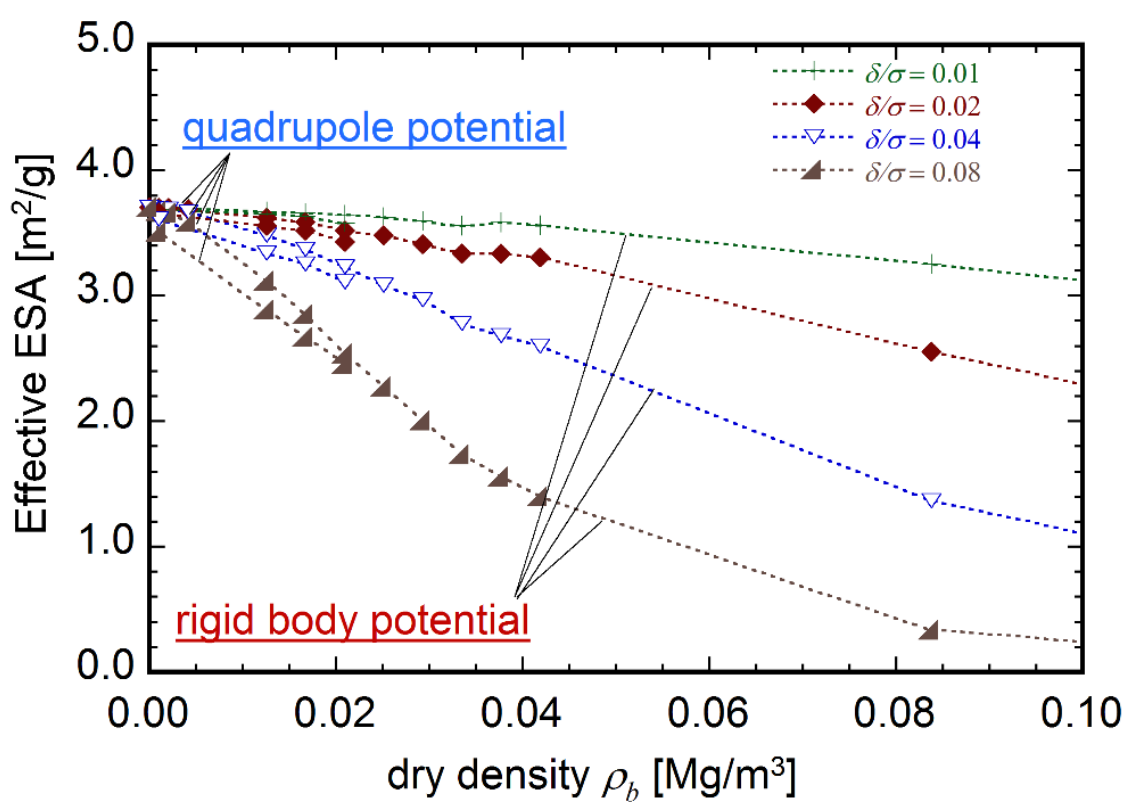

(b) Effective ESA from low to medium density

Fig. 6 Effective ESA calculated from the masking model with various masking parameters $\delta / \sigma$. 


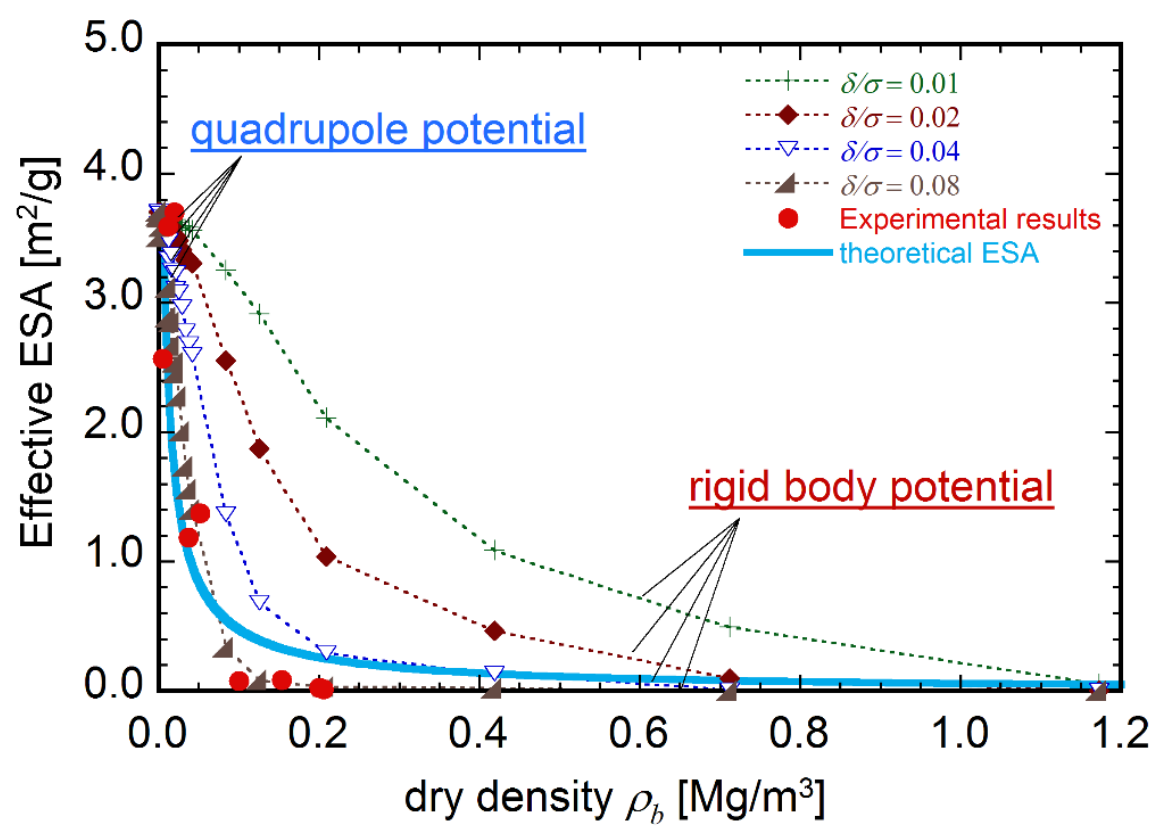

(a) Effective ESA in a wide range of dry densities

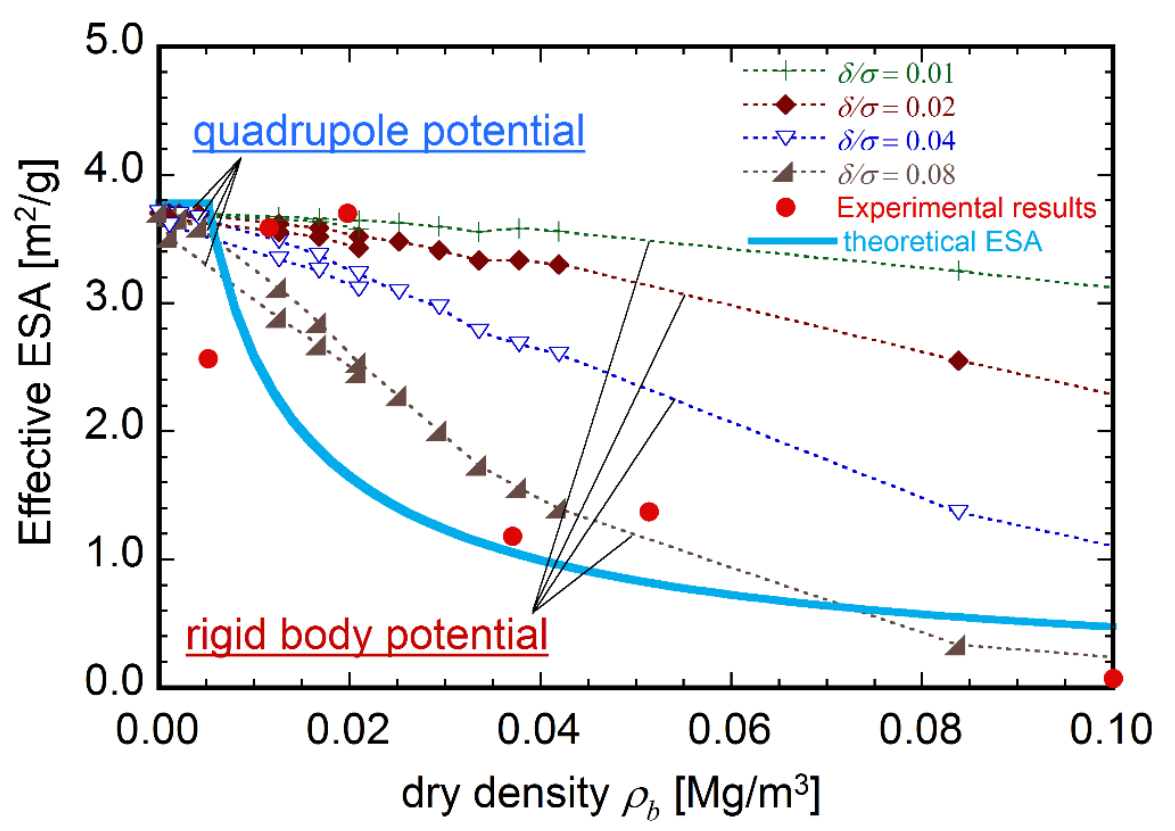

(b) Effective ESA from low to medium density

Fig. 7 Comparison of numerical results of effective ESA, experimental results of dissolution rate and theoretical model under various density conditions. 


\section{Supplementary Table}

Table 1 Results of the dissolution experiment of montmorillonite by AFM

\begin{tabular}{|c|c|c|c|c|c|c|c|c|c|c|}
\hline Sample & 0 & 1 & 2 & 3 & 4 & 5 & 6 & 7 & 2007-c1 & $2007-c 2$ \\
\hline \multirow{2}{*}{ Solution } & \multirow{2}{*}{$\mathrm{H}_{2} \mathrm{O}$} & $0.3 \mathrm{M}$ & $0.3 \mathrm{M}$ & $0.3 \mathrm{M}$ & $0.3 \mathrm{M}$ & $0.3 \mathrm{M}$ & $0.3 \mathrm{M}$ & $0.3 \mathrm{M}$ & $0.3 \mathrm{M}$ & $0.3 \mathrm{M}$ \\
\hline & & $\mathrm{NaOH}$ & $\mathrm{NaOH}$ & $\mathrm{NaOH}$ & $\mathrm{NaOH}$ & $\mathrm{NaOH}$ & $\mathrm{NaOH}$ & $\mathrm{NaOH}$ & $\mathrm{NaOH}$ & $\mathrm{NaOH}$ \\
\hline Volume [ml] & 6.0 & 7.0 & 5.0 & 6.0 & 5.0 & 4.0 & 4.0 & 4.0 & & \\
\hline $\mathrm{KP}[\mathrm{mg}]$ & 1.00 & 34.78 & 55.80 & 118.05 & 174.49 & 200.75 & 691.17 & 965.25 & & \\
\hline Gel volume [ml] & 6.00 & 6.76 & 4.84 & 5.98 & 4.71 & 3.91 & 4.50 & 4.70 & & \\
\hline Density calc. $[\mathrm{g} / \mathrm{ml}]$ & 0.00017 & 0.005 & 0.011 & 0.020 & 0.035 & 0.050 & 0.173 & 0.241 & & \\
\hline Density obs. [g/ml] & 0.00017 & 0.005 & 0.012 & 0.020 & 0.037 & 0.051 & 0.154 & 0.205 & 0.1 & 0.2 \\
\hline Temperature $\left[{ }^{\circ} \mathrm{C}\right]$ & 25 & 70 & 70 & 70 & 70 & 70 & 70 & 70 & 70 & 70 \\
\hline Duration [min] & 0 & 925 & 925 & 925 & 925 & 925 & 990 & 990 & & \\
\hline $\mathrm{ESA}\left[\mathrm{nm}^{2}\right]$ & 773.636 & 652.746 & 581.801 & 566.981 & 689.376 & 738.859 & 756.619 & 759.926 & & \\
\hline $\mathrm{BSA}\left[\mathrm{nm}^{2}\right]$ & 68176.270 & 45593.262 & 40039.062 & 39916.992 & 57128.906 & 54504.395 & 67260.742 & 68054.199 & & \\
\hline $\mathrm{TSA}\left[\mathrm{nm}^{2}\right]$ & 68948.615 & 46221.931 & 40693.226 & 40498.769 & 57872.221 & 55286.785 & 67580.734 & 68828.243 & & \\
\hline Diameter [nm] & 208.331 & 170.367 & 159.655 & 159.411 & 190.708 & 186.275 & 206.929 & 208.145 & & \\
\hline Size median $[\mathrm{nm}]$ & 225.481 & 184.735 & 169.713 & 169.295 & 198.665 & 200.414 & 221.11 & 218.778 & & \\
\hline Volume $\left[\mathrm{nm}^{3}\right]$ & $3.238 \mathrm{E}+10$ & $2.166 \mathrm{E}+10$ & $1.902 \mathrm{E}+10$ & $1.896 \mathrm{E}+10$ & $2.714 \mathrm{E}+10$ & $2.589 \mathrm{E}+10$ & $3.195 \mathrm{E}+10$ & $3.233 \mathrm{E}+10$ & & \\
\hline Rate $\left[\mathrm{mol} / \mathrm{m}^{2} / \mathrm{s}\right]$ & $0.000 \mathrm{E}+00$ & $1.085 \mathrm{E}-09$ & $1.517 \mathrm{E}-09$ & $1.564 \mathrm{E}-09$ & $5.027 \mathrm{E}-10$ & $5.805 \mathrm{E}-10$ & $3.547 \mathrm{E}-11$ & $4.708 \mathrm{E}-12$ & $3.160 \mathrm{E}-11$ & $1.000 \mathrm{E}-11$ \\
\hline
\end{tabular}

